\title{
Slaves no longer: review on role assignment for human-robot joint motor action
}

\author{
Nathanaël Jarrassé ${ }^{1,2}$, Vittorio Sanguineti ${ }^{3}$, and Etienne Burdet ${ }^{1}$.
}

\section{REVIEW PAPER}

\begin{abstract}
This paper summarises findings on the growing field of role assignment policies for human-robot motor interaction. This topic has been investigated by researchers in the psychological theory of joint action, in human intention detection, force control, human-human physical interaction, as well as roboticists interested in developing robots with capabilities for efficient motor interaction with humans. Our goal is to promote fruitful interaction between these distinct communities by: $i$ ) examining the role assignment policies for human-robot joint motor action in experimental psychology and robotics studies, and ii) informing researchers in human-human interaction on existing work in the robotic field. After an overview of roles assignment in current robotic assistants, this paper examines key results about shared control between a robot and a human performing interactive motor tasks. Research on motor interaction between two humans has inspired recent developments that may extend the use of robots to applications requiring continuous mechanical interaction with humans.
\end{abstract}

Index Terms-motor joint action, physical human-robot interaction (pHRI), human-human interaction, role assignment policies, master-slave, education

\section{INTRODUCTION}

Many common tasks, such as sawing, dancing, physical rehabilitation, fighting, mating, carrying a table together (Fig.1), rely on the motor interaction of two humans. Here "motor interaction" describes any interaction with the environment, a robot or a human, involving a sensorimotor exchange. We preferred this expression to the commonly used "physical interaction" and "haptic interaction", because physics is not restricted to mechanics, and haptics focuses on (touch and force) sensing rather than motor action." While we have some knowledge of how humans adapt to passive or active environments (e.g. [Franklin et al., 2008]), how humans control motor interaction with peers is still largely unknown. Understanding how humans collaborate in tasks requiring motor interaction is not only an interesting and challenging new field of research, but may also be crucial in the design of robots

\footnotetext{
1 Department of Bioengineering, Imperial College of Science, Technology and Medicine, London, United Kingdom.

${ }^{2}$ Institute of Intelligent Systems and Robotics (ISIR), UPMC Univ. Paris 06, CNRS - UMR 7222, F-75005, Paris, France.

3 Department of Informatics, Bioengineering, Robotics and Systems Engineering, University of Genoa, Italy.

Corresponding author:

Nathanaël Jarrassé, Department of Bioengineering, RSM 4.28, Royal Schoo of Mines, Imperial College London, London SW7 2AZ, United Kingdom. Email: $\{$ n.jarrasse, e.burdet $\} @$ imperial.ac.uk
}

interacting with humans (physical human-robot interaction, pHRI).

Recent years have seen the appearance of 'assistive robots', including assistive devices for manufacturing [Akella et al., 1999], [Schraft et al., 2005], robotic systems for teleoperation [Hokayem and Spong, 2006], assisted driving systems increasingly included in cars [Gietelink et al., 2006], robotic wheelchairs to increase the mobility of people with physical or cognitive deficits [Zeng et al., 2009], workstations with haptic feedback that can be used to train surgeons [Nudehi et al., 2005], robotic exoskeletons to increase the user's force capabilities [Kazerooni, 1990], and rehabilitation robots to increase the amount and intensity of physical therapy after stroke [Kwakkel et al., 2008].
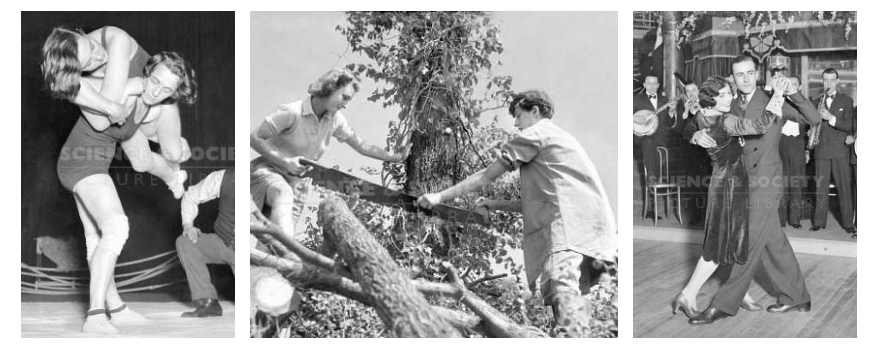

Fig. 1. Wrestling, sawing, dancing involve specific roles for the two partners (C)Kodak Collection-NMeM-Science Society, Picture Library)

These applications demand a continuous, or at least a prolonged period of physical interaction between a robot and its human user toward a common goal, during which they use either similar or complementary roles. In some cases these roles are established a priori, as a direct consequence of the nature of the task [Jarrassé et al., 2012]. In other situations, the same task allows for a variety of role assignments: For example, two subjects carrying a heavy table could collaborate as equal partners, cooperate as a master-slave dyad (i.e. one subject would provide most of the workforce under the other's partner supervision), or even work as competitors if each wants to bring the table to a different location. In such tasks, it would robots with the capability to negotiate and adapt their own role to the overall goal and the behavior of their human partner. The capability to understand human motor behavior and adapt its role in completing a motor task completion is probably the key to developing versatile interactive robots.

Knowing how humans control motor interaction with a partner may aid in the design of efficient human-robot interaction strategies. Furthermore, a robot adapting his role and action the same way a human partner do may be more 
intuitive to a human operator, thus requiring less effort during use. Finally, a robot able to learn from collaboration with a skilled human worker would enable industrial manufacturing companies to move the robot assistant from one workstation to another without extensive reprogramming, in contrast to current industrial robots [Krüger et al., 2009]. In summary, a deeper understanding of the motor interaction between humans or between a robot and a human could widely broaden the scope of robot applications of robots [Santis et al., 2008].

This paper first provides an overview of simple roles given to robotic assistants based on master-slave control and on the decomposition of tasks into distinct actions. Humanhuman interaction investigations demonstrating roles of greater complexity are then reviewed. Recent key studies about role assignment policies for improved human-robot joint action are then presented, starting with investigations on human motor joint action, followed by with new approaches to improve physical human-robot interaction in collaborative actions. Finally, the paper discusses future trends in the field of motor interaction between robots and humans.

\section{COllaborative ACTIONS IN PHRI}

\section{A. Existing taxonomies, classifications and roles definitions}

The ways through which humans and robots interact with each other have been described in general taxonomies stemming from the field of human-computer interaction (HCi) [Agah and Tanie, 1999], [Yanco and Drury, 2002]. However, these taxonomies do not specifically address physical humanrobot interaction (pHRi) [Yanco and Drury, 2004].

On the other hand, existing descriptions of the different motor interaction schemes and consequentially difficult to utilize in other applications. For example, [Burghart et al., 2002] defined a classification of role distribution schemes between human and robot that is dedicated to the different kinds of joint actions (with or without a tool) performed with humanoid robots. Though interesting, this approach lacks quantitative and exhaustive analysis of interaction parameters along with a rigorous consideration of the observation timescale, which may lead to misinterpretations. For example, considering a small window of time when analyzing the exchanges between subjects can suggest a role repartition that is not representative of the entire task.

As a consequence, studies that have classified possible role distributions are not used practically because of their lack of formal definitions. For instance, [Ong et al., 2008] identified five human-robot relationships based mainly on teleoperation theories [Sheridan, 1992]: master-slave, supervisorsubordinate, partner-partner, teacher-learner and fully autonomous robot. This framework can be used as a high-level description of the interaction, but not for a detailed analysis, as the relationships between the interacting agents arise from different field/cases (e.g. teleoperation, high level supervision, learning by demonstration) and would need a quantitative analysis of role strategies. Many studies have been performed in the field of teleoperation (which addresses the problem of remote physical interaction) on task-specific controllers for haptic assistance and passive/active guidance of the operator (as reviewed in [Passenberg et al., 2010]) which are based on simple fixed asymmetric role distributions between human and robot.
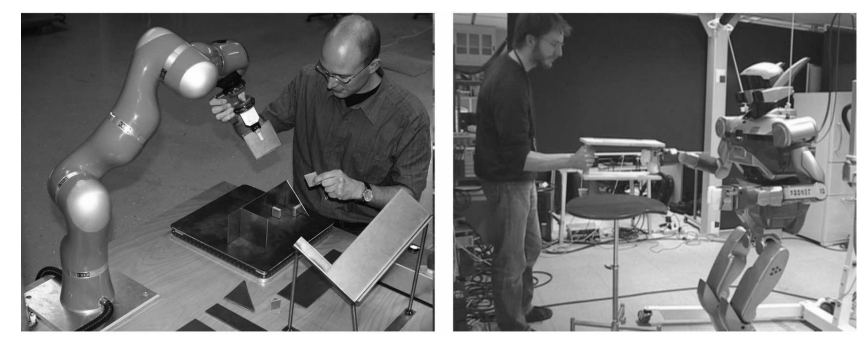

Fig. 2. Human-Robot joint motor action. Left: LWR robot from DLR. Right: HRP2 robot used in [Evrard and Kheddar, 2009a].

The lack of precise terminology on the distribution of roles in motor joint action schemes stems from the complexity of understanding and explaining physical interaction during a task. The multimodal exchange between the partners increases the complexity of the interaction drastically, and an appropriate analysis of this interaction must consider both the energy to physically perform the task and the information used to advise partners about the ongoing action. A taxonomy of roles in motor interactions hat both describe and generate controllers for such interactions has recently been introduced [Jarrassé et al., 2012]. This framework, however, awaits to be applied in order to test its utility and describe the specific multimodal exchanges on which transitions are based.

Motor interaction involves potential hazards because of the direct contact and energy exchange. Robotic assistants (Fig. 2) are usually designed to produce forces whose magnitude may be as large or even larger than those exerted by humans. Thus, they can cause severe injuries to their human partners. This may explain why human-robot interaction has often been treated as a strict asymmetric master-slave relationship, and why much recent work has focused on ensuring safety [De Luca et al., 2006], [Haddadin et al., 2008] rather than on increasing the autonomy of robotic partners.

\section{B. Basic mechanisms: Impedance control and prediction ca- pabilities}

Recent works has shown how force and mechanical impedance (the response to an imposed motion perturbation) are adapted in humans [Burdet et al., 2001], [Franklin et al., 2003] in response to specific types of dynamic environments. Similar mechanisms can be implemented in robots [Yang et al., 2011] to ensure stable and efficient performance with minimal effort. Impedance control allows allows for specification of the dynamic relationship between the position and the exerted force [Hogan, 1985], [Kazerooni et al., 1986].

In robots, impedance control has often been used to deal with environments of unknown or varying mechanical properties [Colgate and Hogan, 1989]. Important results were obtained on interaction stability despite force and position signals discretization [Miller et al., 2000], [Adams and Hannaford, 1999], or in complex tasks like 
assembly [Surdilovic et al., 2001]. In rehabilitation robots, impedance control is used to implement specific forms of interaction (e.g. active assistance or active resistance) [Marchal-Crespo and Reinkensmeyer, 2009]. In all these cases, the respective 'roles' of the robot and the human are established a priori. In general, approaches based on impedance control consider an interacting human as a 'perturbation'. This limits the supported forms of humanrobot interaction to situations in which the robot 'leads' the movement.

Another approach to improve the motor exchanges between human and robots consists of providing robots with an ability to predict human intention, for example by using gaze tracking [Sakita et al., 2004] or by recognizing characteristic patterns according to human behavior models [Pentland and Liu, 1999], and reacting or adapting its behavior accordingly. However, the versatility of human interactive behaviors makes them inherently difficult to predict. Models that consider the different communication channels (language, gesture, conscious and unconscious behaviors, etc.) are thus complex [Sato et al., 1994] and can only be used for simple interaction scenarios with a small number of possible strategies.

While particular role distributions may emerge from these conventional control strategies for human-robot motor interaction, they do not consider the high-level role assignment issues which are required to deal with the complexity of many collaborative tasks to which a robot could contribute. This will be the main focus for the remainder of this review.

\section{Roles assignment in human-robot motor interaction}

1) Robotic slaves: In the area of human-robot motor interaction, the master-slave scheme refers generally to the form of interaction where a human (master) generates commands that the interacting robot (slave) executes. Thus the master-slave scheme corresponds to an asymmetric relationship in which only the master makes decisions, and role distribution is not questioned.
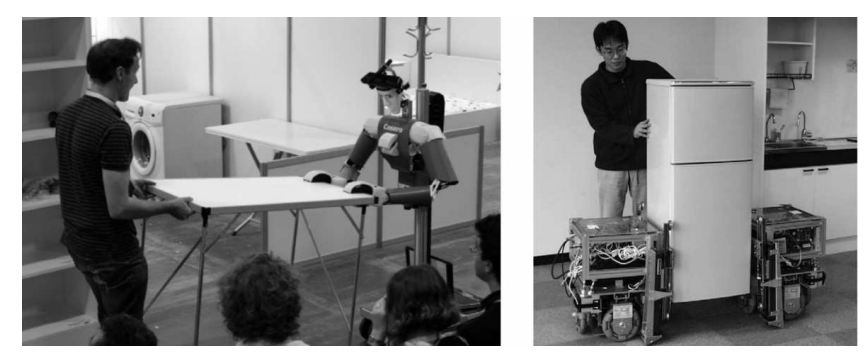

Fig. 3. Master-slave examples. Left: Cosero [Stückler and S Behnke, 2011]. Right: Dr Helper [Kosuge and Hirata, 2004].

An important number of studies have been performed on robotic slaves to assist humans in performing tasks, in particular for lifting and carrying heavy or bulky objects. Various platforms, such as mobile robots with a robotic arm, have been developed [Kosuge and Hirata, 2004], which are equipped with controllers to detect the intentions of the human operator [Maeda et al., 2001], [Yokoyama et al., 2003],
[Wojtara et al., 2009], [Stückler and S Behnke, 2011] or to manage multiple slave robots [Kosuge et al., 1994]. The "leader/follower" concept found in many studies, in which only the follower adapts its movements to synchronize with the leader, is similar to the "master/slave" configuration. For example in [Stückler and S Behnke, 2011], the robot follows the human guidance during a cooperative table lifting task by tracking the movement of his hands holding the table.

An application of this asymmetric role assignment is common in exoskeletons conceived for the purpose of amplifying the physical capabilities of humans [Kazerooni, 1990], [Kazerooni and Guo, 1993]. Several such force extender exoskeletons have been developed in recent years, in particular for military applications [Dollar and Herr, 2008], [Kazerooni et al., 2005]. Here, the robot is designed to minimize the human master effort, while it is mechanically connected to the human body and transferring power to it.

Robotic slaves also encompass systems to provide forces or trajectory corrections [Khatib, 1999], or to guide movements within a restricted workspace [Peshkin and Colgate, 2001], [Zeng et al., 2009]. Robotic aids that guide the user's motion along desired directions while preventing motion in undesired directions or regions of the workspace through 'virtual fixtures' [Rosenberg, 1993] can be considered slaves because they cannot complete the main task alone and exists only to provide support during action. Such robotic aids are known as intelligent assistive devices (IAD). Despite their name, the collaborative robots or cobots described in [Colgate et al., 1996] do not collaborate as an equal partner would, but implement a master-slave behavior. Cobots track human operator behavior and react accordingly, for example in [Colgate et al., 2003] a load lifting device provides assistance according to the angular movements of the loading cable. Therefore these assistive devices can be considered slaves.

Finally, robot teach pendants where the human teacher directly moves the robot that records the motion to reproduce it, or imitation learning [Pastor et al., 2011] (where the robot is moved according to recorded data of human movement), also correspond to a master-slave scheme. Indeed, in these cases, the robot is passively following the human teacher, at least during the learning period.

2) Master-slave vs. co-activity: Distinct from the masterslave scheme are divisible tasks, where robot or/and human agents interact without needing each to to know what the other is doing, and incidentally interact and succeed in the common task through co-activity. Separating tasks in independent but complementary subtasks where each agent performs well often is an efficient way to carry out 'joint' action: neither sensory exchange nor negotiation is required, enabling simple solutions without inference. Such situations typically arise when the task is decomposed into subtasks carried out by independent controllers. An example is the Acrobot robot assistant for bone surgery [Cobb et al., 2006], which constrains the surgeon's motion to a predefined region, facilitating surgery without requiring knowledge of the surgical task. Similarly, simple assistive devices developed to help manufacturing, e.g. by compensating gravity using springs during tool or parts manipulation (and which are not reacting according to any 
human worker action), cannot be considered slaves because no information exchange is needed, as the worker and robot complete separate actions.

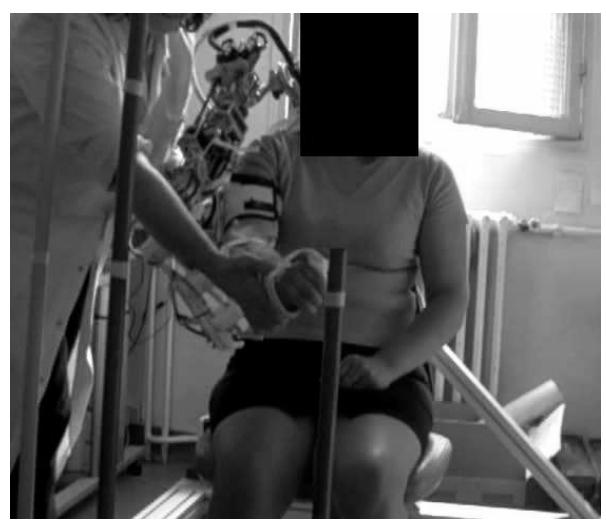

Fig. 4. Arm manipulation of an hemiplegic patient by a physical therapist and an upper-limb robotic exoskeleton, performed at the Raymon Poincaré Hospital, Garches, France. Such tasks require a complex sensorimotor coordination strategy between the physiotherapist and the training patient.

3) Advanced forms of interaction: While division in independent subtasks may facilitate relatively stereotyped actions, complex tasks would benefit from a more sophisticated sharing between the human and robot [Sheridan, 1997]. For instance, neuro-rehabilitation (Fig. 4), in which robotic devices and their controllers assist patients to develop their movement capabilities [Hogan and Krebs, 2004], requires task sharing beyond pure master-slave roles. For example, successful neuromotor rehabilitation requires a therapist to assist a (e.g. post-stroke) patient in moving the arm while inferring her or his sensorimotor state and tuning motion assistance correspondingly in order to help the patient actively working on improving her or his capabilities. Similarly, the growing field of smart/robotic wheelchairs would benefit from collaborative control strategies and shared control policies letting the user take charge of the overall control of the wheelchair but assisting her or him manoeuvring [Zeng et al., 2009].

\section{RESULTS FROM HUMAN-HUMAN INTERACTION}

The presence of multiple actuators and decision centers makes the control of joint motor actions complex. Following pioneering work in the psychology of joint action, research in the field of human-human interaction (HHI) has investigated the control of motor interaction between humans. These studies highlighted the potential of collaboration and generated enthusiasm in the HRI field, leading to controllers overriding the rigid schemes and the trick of dividing task into independent subtasks. However this field is relatively young and has so far led to few real applications.

\section{A. From human-human to human-robot interaction}

1) Psychological and social aspects of HHI: Psychological studies have provided some evidence for the benefits of interaction between humans, e.g. [Sebanz et al., 2003]: The observation and knowledge about the partner's action affects one's own actions even when an actual coordination is not required, exhibiting "motor resonance." Relations between perceptual judgements about the partner's actions and the current state of one's own motor system were also identified [Schütz-Bosbach and Prinz, 2007], showing that high level cognitive processes influence the joint action scenario and the role distribution between partners (through multi-modal exchanges) and affect the interactive performance.

The control of joint actions involves high-level cognitive mechanisms to feel, evaluate and understand the partner's intentions and actions [Sebanz et al., 2006]. In psychology, the tendency for healthy adults to automatically impute mental states to oneself and others is usually referred as Theory of Mind (ToM) [Premack and Woodruff, 1978]. ToM provides subjects with an ability to make inferences about others by attributing beliefs, feelings, desires and intentions to oneself and others, and is believed to be essential for social and physical interactions [Sebanz et al., 2003]. To perform efficient joint actions, subjects are required to emulate an internal model of the partner or of its influence on the shared task.

Other psychological phenomena considered to be factors of interactive behaviors between humans [Knoblich et al., 2010], [Obhi and Sebanz, 2011] include co-representation mechanisms, such as simulation theory [Gallese and Goldman, 1998] (mental projection in which one subject temporarily adopts the partner's point of view), social facilitation [Zajonc, 1965] (tendency for people to perform tasks in a better way when other people are considering his/her action), and Interpersonal coordination mechanisms such as mimicry [Chartrand and Bargh, 1999] (unconscious tendency of subject to synchronize and mimic other behavior or gesture, generally to facilitate acceptation). Related psychological theories are synchrony [Richardson et al., 2007] (tendency of subjects to synchronize their actions), game theory [Myerson, 1997] (a mathematical approach to model strategic situations in which an individual's success in making choices depends on the choices of others), and the theory of affordance [Gibson et al., 1977] (stating that the world - object or even human partner - is perceived in terms of object possibilities).

2) HRI to investigate HHI: Several studies on joint action were conducted to examine the information transfer for joint action between two humans. The analysis of exchanged signals between subjects and some teleoperated task simulators, suggest that force feedback [Glynn et al., 2001] allows people to perform better in a joint task than when performing alone if haptic and visual feedback are well synchronized. Studies in which two partners manipulated the same virtual object showed that haptic feedback leads to improvements in task performance [Sallnas, 2001], [Groten et al., 2010]. Haptic feedback is also crucial for action coordination in more complex tasks such as dancing [Gentry, 2005] as well as for dynamic role division [Pham et al., 2010]. These studies further suggested that even when a haptic communication is established and negotiation phases are observed [Groten et al., 2010], parameters of the role distribution such as the dominance behavior appear to be more linked to the subjects' nature than to interaction's parameters [Groten et al., 2009].

[Reed et al., 2006a], [Reed and Peshkin, 2008] conducted 
experiments to investigate haptic joint action with continuous physical contact between two partners. The partners were connected by a two-handled crank mounted on a controlled directdrive motor. This motor could measure and interact with the common circular movement, and the applied force measured at each handle could be used to test interaction strategies. The authors demonstrated that subjects perform point-to-point movements faster connected than alone [Reed et al., 2006b]. They also suggested that some dyads (i.e. pairs of partners) adopt specialised roles, where one partner is in charge of the acceleration and the other controls the braking to reach the right position [Reed and Peshkin, 2008], which might explain the benefits from [Reed et al., 2006a]. This pioneering work, however, lacks quantitative evidence of role specialization, and simple modelling implemented on the robot did not succeed in providing the benefits observed with a human partner [Reed et al., 2007]. [Ueha et al., 2009] extended these experiments and accurately defined the human dynamical role division and control by using an additional degree of force measurement (due to the use of external force sensors placed below each handle). These studies have drawn attention to the potential of motor interaction and stimulated further research in this area.

\section{B. Roles switching: a key to partner's equality?}

While the interaction between two agents may be designed as an equalitarian and unconstrained relationship as is believed to be used by humans, typical human-human interaction may correspond more to an asymmetric scheme with multiple switchings between roles. Several studies were thus conducted to try identify and understand the switching processes between strategies for collaboration.

[Stefanov et al., 2009] studied interaction during a tracking task and defined a tri-state logic composed of two roles and one "no behavior" condition based on the signs of the force, velocity and acceleration. A role distribution similar to a leader-follower combination involves a "conductor" who decides what the system should do and expresses this intention via haptic signals (and through energy dissipation), and an "executor" who performs the action as determined by the conductor (thus injecting energy). This approach is interesting as it bypasses the global rigidity of conventional fixed asymmetric relationship, by allowing multiple role switchings (changes of the direction of the asymmetry) during the completion of the task, and by letting the executor participate in the task. It gives an interesting insight about low-level interaction, though the fine temporal resolution and the association of multiple roles to each partner make it difficult to interpret the results. Preliminary work on using such classification in teleoperation has recently been presented in [Corredor and Sofrony, 2011].

Nevertheless, in light of several considerations from studies on haptic communication, the "role switching" phase could potentially be an episodic and preliminary negotiation phase that would disappear when task is performed repeatedly by the same partners.

\section{Consideration of mechanical impedance}

While all experiments presented above considered only the kinematics and forces, a recent study [Melendez-Calderon et al., 2011] also investigated how human dyads control impedance, which is important to ensure interaction stability and and robust response to perturbations. This work developed automatic identification of the interaction strategies, and showed that dyads formed of naïve subjects that had never met started by a negotiation phase during which roles are switched after which specialization that is specific to a particular dyad and robust to perturbations, occurs[Melendez-Calderon, 2011].

\section{CONTROL SCHEMES TO IMPROVE HUMAN-ROBOT JOINT ACTION}

\section{A. Switching and adapting roles}

Since a few years "equalitarian" roles distribution beyond the master-slave scheme have been investigated such as supervisor-subordinate, cooperators, or teacherlearner [Ong et al., 2008], [Ikemoto et al., 2009], [Pastor et al., 2011]. For example, [Lawitzky et al., 2010] evaluated three different effort sharing policies during transport of a bulky object by a human and a robot: balanced-effort behavior, maximum, and minimum roboteffort behavior. Performances obtained with each of these conditions were evaluated, and results showed an improvement (minimization of applied force level and tracking error) through a more proactive robot behavior which is consistent with previous research on motor interaction.

Besides these new roles, [Evrard and Kheddar, 2009b], [Evrard and Kheddar, 2009a] introduced a flexible role distribution enabling each partner to tune between the two distinct extreme behaviors of leader and follower using a homotopy (a weighting function that allows a continuous change between two behaviors), giving rise to an implicit bilateral coupling [Kheddar, 2011]. With this approach, each partner can claim or give up leadership in a smooth way. While this attractive framework was recently demonstrated on the object lifting between a human and an HRP2 humanoid robot, it does not determine yet how the redundancy of the two interacting partners is solved, i.e. how it could be used to design interaction control in an application. Using the homotopy framework, an experiment was developed in which the lifting of a table between a human a humanoid was analysed in the state space and identified using Gaussian mixture regression [Evrard et al., 2009]. This probabilistic model was then used by the robot in order to switch between the leader and follower behaviors. The robot was able to adapt its behavior to human subjects who changed their role during the task, however the results were not very robust, and lacked agreement with human dyads.

In a similar approach to the homotopy, [Oguz et al., 2010] defined a dynamic role-based effort sharing scheme utilizing a force threshold on a known user force profile to improve interaction quality through role negotiation during a game, in which a ball rolling on a plane must hit several targets. Here, role distribution was restricted to a discrete tri-logic state 
("user dominant," "role blending" and "equal control") and led to no statistically significant improvement in task parameters including completion time, total path length, deviation of the ball from the ideal path, integral of time, and energy spent. Also, ideas for new online policies have been recently proposed in [Passenberg et al., 2011a] where the best assistance that a virtual assistant should exhibit to help a subject minimize error and interaction force during the completion of a $2 \mathrm{D}$ maze task is computed.

Based on a formal analysis of human-robot interaction during a load transport task, [Mortl et al., 2012] defined and evaluated different possibilities for role assignment: two dynamic role exchange mechanisms in which adaptation is based on human force feedback measurement and one generic static role allocation strategy for comparison purposes. The dynamic role allocation strategy parameter is adapted according to the magnitude of the partner's contribution in the redundant direction, defined as the direction where effort sharing between the agents can take place. Role assignment mechanisms were evaluated in a user study on 18 subjects based on both quantitative measures indices (completion time, effort, or amount of disagreement) and qualitative measurement of user experience with a questionnaire. The results showed that a continuous dynamic role assignment policy leads to better performance than a constant role assignment one. However, it seems that humans preferred the constant role, where robot behavior is more predictable and thus easier to consider in their motor action.

Studies on robot adaptive interaction with a human can also be encountered when reproducing human hand-shaking with a robot, an interesting bilateral task. Dedicated robot controllers based on a hidden Markov model approach used to estimate human intentions and adapt robot behavior [Wang et al., 2009], or online adaptation to interaction dynamics [Guanghui et al., 2011] seem to provide realistic experiences.

\section{B. Education schemes}

An important human-robot interaction scheme that tends to be increasingly used is education, where both the human can teach the robot and conversely some robots may be used to teach a human. For instance, the "learning-by-demonstration" approach where a robot learner is actively performing a task and is corrected by the human teacher through motor interaction [Schaal et al., 1997], [Calinon and Billard, 2007], [Lee et al., 2011] is an educational type of interaction, in which a human helps the (humanoid) robot to refine a previously learned movement by kinesthetic teaching.

Similarly, [Ikemoto et al., 2009] developed an algorithm dedicated to robot learning through physical interaction with humans. The efficiency of their method was evaluated in an experiment where a human helps a humanoid robot to stand up and to learn temporal aspects of the postural sequence required to stand up (Fig.5). The implementation results in their works showed that improvements are due to a bilateral learning process that takes place in both partners. Even if only the learner's behavior is described and tuned, these results underline the importance of teacher adaptation to let the subject learn and thus the importance of the bilateral exchange.

On the other hand, a robot may help a human partner to work more precisely, in a more efficient way, with less effort or in a more ergonomic way. For example, in [Boy et al., 2007], a passive mobile robotic platform (cobot) mechanically constrains the motion from a human operator encourages him to learn ergonomic paths, and enables him to position heavy objects more precisely and move them with less effort. The results of [Boy et al., 2007] show that subjects working with this "learning cobot" adopt a more ergonomic behavior minimizing the back torsion. We note that in this scheme learning occurs on both sides, as the robot guiding path could be adapted to the changes in the environment or of the human strategy.

Even if the passive mode used for the first stage of robotic rehabilitation is similar to a raw master-slave, the active mode giving assistance as needed to encourage patient involvement in the task is similar to such education scheme [Lum, 2002]. This can be realized by simultaneously relaxing assistance and satisfying performance [Emken and Reinkensmeyer, 2005], [Franklin et al., 2008]. The robot gradually minimizes its involvement in the task completion to encourage human participation and accelerate motor skill learning [Reinkensmeyer and Patton, 2009].

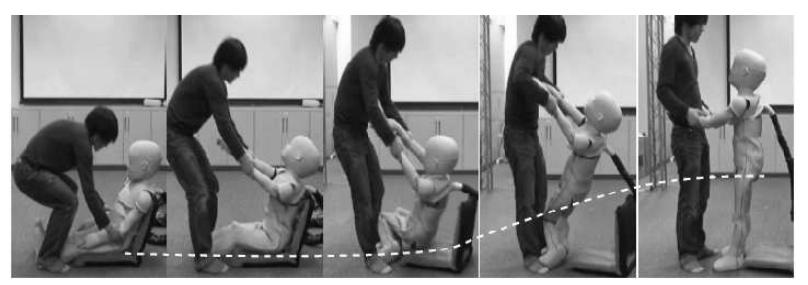

Fig. 5. Kinaesthetic teaching of a standing-up task [Ikemoto et al., 2009].

\section{Discussion}

The area covered by published works on motor interaction between human and robot include the classification of interactive motor behaviors [Yanco and Drury, 2004], [Burghart et al., 2002], [Ong et al., 2008], [Jarrassé et al., 2012], observation and understanding of role distribution in human dyads [Reed et al., 2007], [Reed and Peshkin, 2008], [Stefanov et al., 2009], [Melendez-Calderon et al., 2011], attempts of replicating these interaction kinds with robots [Reed and Peshkin, 2008], [Ueha et al., 2009], [Lawitzky et al., 2010], and controllers able to modify the roles during the interaction [Evrard and Kheddar, 2009b], [Evrard et al., 2009], [Oguz et al., 2010],

[Passenberg et al., 2011a], [Corredor and Sofrony, 2011], [Mortl et al., 2012]. While a large number of papers call for more flexibility than the master-slave scheme, only a few works so far attempted to gain a deep understanding of the physical interaction issues or implement even simple collaborative behaviors.

Table I groups studies providing control schemes beyond master-slave. A common goal consists of developing robot assistants capable of collaborating with a human partner rather 


\begin{tabular}{|l|l|l|}
\hline Strategy & Task & Existing work \\
\hline \multirow{2}{*}{ divisible task assistant } & load lifting assistance & [Colgate et al., 1996], [Peshkin and Colgate, 2001], [Colgate et al., 2003] \\
\cline { 2 - 3 } & motion assistance & [Rosenberg, 1993] \\
\hline \multirow{2}{*}{ fixed assymetric roles } & manipulation assistance & [Ueha et al., 2009], [Lawitzky et al., 2010] \\
\cline { 2 - 3 } & kinesthetic teaching & [Pastor et al., 2011], [Ikemoto et al., 2009] \\
\hline \multirow{2}{*}{ switching roles } & load lifting assistance & [Evrard et al., 2009], [Evrard and Kheddar, 2009b] \\
\cline { 2 - 3 } & haptic guidance & [Oguz et al., 2010], [Passenberg et al., 2011a], [Corredor and Sofrony, 2011], [Mortl et al., 2012] \\
\cline { 2 - 3 } & hand-shaking partner & [Wang et al., 2009], [Guanghui et al., 2011] \\
\hline
\end{tabular}

TABLE I

COLLABORATIVE ROBOTS BEYOND THE MASTER-SLAVE SCHEME.

than simply cooperating with her or him. In a collaboration, there is no a priori role distribution, but a spontaneous role distribution depending on the interaction history and mutual "online" adaptation. In contrast, cooperation occurs when different roles are ascribed to the agents prior to the beginning of a task, and this distribution is not questioned until its completion [Dillenbourg et al., 1996].

We believe that the following main issues need to be addressed in order to develop robotic systems capable of true collaboration:

- A categorization of role attribution in joint motor action based on multisensory cues should be developed. While research in computational neuroscience commonly analyzes full motion control involving complex coordination and energy consumption, in most studies in human-robot motor interaction the analysis is limited to interaction forces [Groten et al., 2009], [Groten et al., 2010].

- There is an increasing number of studies on psychological and social factors affecting joint actions [Sebanz et al., 2006], [Chartrand and Bargh, 1999], while most robotic studies have focussed on kinematic or force information exchanges, and neglected other cues which are necessary to understand the switching between roles. Preliminary discussions on partner perception such as [Reed and Peshkin, 2008] are worth pursuing. Also, physiological parameters such as heart rate [Damen and Brunia, 1987], gaze patterns [Vertegaal et al., 2001], facial expression [Breazeal et al., 2004], may be considered as a mean of inferring a human's state and thus to select interaction roles.

- Interactive behaviors such as the competition between actors, which are currently under-studied, should be investigated and used. In some cases competition may produce more efficient performance at the dyad's level than positive collaborations. For example, [Passenberg et al., 2011a] presents preliminary work on which best strategy the robot should exhibit if the interacting subject agrees or not on the motion to perform.

- Mechanical impedance is a main determinant of interactions, and should thus be considered in human-human and human-robot motor interaction. Modern torque controlled robots and the development of Variable Impedance Actuators (VIA) [Bicchi et al., 2005] allow implementation of sensitive impedance control strategies. Recent studies introduced human-like concurrent adaptation of trajectory, force and impedance [Franklin et al., 2008], [Burdet et al., 2010] on the basis of which efficient interaction schemes may be developed.

- As explained above, collaboration may be achieved through switching of asymmetric relations, yielding a symmetric relation overall. Research has so far considered interaction only at the local level, and it is necessary to consider the interactive behavior at the global level. This requires the development of generic (adaptive) controllers suitable for various tasks. In contrast, existing controllers with adaptive behavior [Evrard and Kheddar, 2009b], [Oguz et al., 2010] are dedicated to a specific task. A few studies [Passenberg et al., 2011a], [Passenberg et al., 2011b] have started to identify behavior selection for a robotic assistant.

- Finally, the psychological aspects of the joint action may be a key to a deep understanding of the motor joint action and coordination strategies. Indeed, motor interaction between humans involves both physical and psychological factors. When a human is collaborating with a partner, s/he tends to analyse the partner's reaction, understand the partner's action, and use this information together with previously learned knowledge to adapt her or his behavior and strategy. While roboticists focused on the sensorimotor exchanges, psychologists have revealed cognitive processes occurring during collaboration. Pioneering experimental psychologists have begun to build a bridge to robotic studies [Sebanz et al., 2003], [Sebanz et al., 2006], and their research results could be considered for future robot control design. Taking these phenomena into account could both clarify some of the observed phenomena (by limiting the effects of cognition), and provide quantitative results to psychologists enabling them to test and refine their theories.

\section{ACKNOWLEDGMENT}

We thank Wayne Dailey for editing our text. This study was supported in part by the EU FP7-ICT-231724 HUMOUR grant.

\section{REFERENCES}

[Adams and Hannaford, 1999] Adams, R. and Hannaford, B. (1999). Stable haptic interaction with virtual environments. IEEE Transactions on Robotics and Automation, 15(3):465-474.

[Agah and Tanie, 1999] Agah, A. and Tanie, K. (1999). Taxonomy of research on human interactions with intelligent systems. Proceedings of the IEEE International Conference on Systems, Man, and Cybernetics, pages 965-970. 
[Akella et al., 1999] Akella, P., Peshkin, M., Colgate, E., Wannasuphoprasit, W., Nagesh, N., Wells, J., Holland, S., Pearson, T., and Peacock, B. (1999). Cobots for the automobile assembly line. In Proceedings of the IEEE International Conference on Robotics \& Automation (ICRA), volume 1, pages $728-733$.

[Bicchi et al., 2005] Bicchi, A., Tonietti, G., Bavaro, M., and Piccigallo, M. (2005). Variable stiffness actuators for fast and safe motion control. In Dario, P. and Chatila, R., editors, Robotics Research, volume 15 of Springer Tracts in Advanced Robotics, pages 527-536. Springer Berlin / Heidelberg.

[Boy et al., 2007] Boy, E. S., Burdet, E., Teo, C. L., and Colgate, J. E. (2007). Investigation of motion guidance with scooter cobot and collaborative learning. Robotics, IEEE Transactions on, 23(2):245-255.

[Breazeal et al., 2004] Breazeal, C., Hoffman, G., and Lockerd, A. (2004). Teaching and working with robots as a collaboration. In Proceedings of the International Joint Conference on Autonomous Agents and Multiagent Systems, pages 1030-1037.

[Burdet et al., 2010] Burdet, E., Ganesh, G., Yang, C., and Albu-Schaeffer, A. (2010). Learning Interaction Force, Impedance and Trajectory: by Humans, for Robots. In Proceedings of International Symposium on Experimental Robotics.

[Burdet et al., 2001] Burdet, E., Osu, R., Franklin, D., Milner, T., and Kawato, M. (2001). The central nervous system stabilizes unstable dynamics by learning optimal impedance. Nature, 414(6862):446-449.

[Burghart et al., 2002] Burghart, C., Yigit, S., Kerpa, O., Osswald, D., and Woern, H. (2002). Concept for human robot co-operation integrating artificial haptic perception. Intelligent Autonomous Systems.

[Calinon and Billard, 2007] Calinon, S. and Billard, A. (2007). Incremental learning of gestures by imitation in a humanoid robot. Proceeding of the ACM/IEEE International Conference on Human-Robot Interaction (HRI), page 255.

[Chartrand and Bargh, 1999] Chartrand, T. and Bargh, J. (1999). The chameleon effect: The perception-behavior link and social interaction Journal of personality and social psychology, 76(6):893.

[Cobb et al., 2006] Cobb, J., Henckel, J., Gomes, P., Harris, S., Jakopec, M., Rodriguez, F., Barrett, A., and Davies, B. (2006). Hands-on robotic unicompartmental knee replacement: a prospective, randomised controlled study of the acrobot system. Journal of Bone and Joint Surgery-British Volume, 88(2): 188

[Colgate et al., 1996] Colgate, J., Edward, J., and Peshkin, M. (1996) Cobots: robots for collaboration with human operators. In Proceedings of the International Mechanical Engineering Congress and Exhibition.

[Colgate and Hogan, 1989] Colgate, J. and Hogan, N. (1989). An analysis of contact instability in terms of passive physical equivalents. In Proceedings of the IEEE International Conference on Robotics \& Automation (ICRA), pages $404-409$.

[Colgate et al., 2003] Colgate, J., Peshkin, M., and Klostermeyer, S. (2003). Intelligent assist devices in industrial applications: a review. Proceedings of the IEEE/RSJ International Conference on Intelligent Robots and Systems (IROS), pages 2516-2521.

[Corredor and Sofrony, 2011] Corredor, J. and Sofrony, J. (2011). Shared control based on roles for telerobotic systems. In Proceedings of the IEEE Latin American Colombian Conference on Automatic Control and Industry Applications (LARC), pages 1-6.

[Damen and Brunia, 1987] Damen, E. and Brunia, C. (1987). Changes in Heart Rate and Slow Brain Potentials Related to Motor Preparation and Stimulus Anticipation in a Time Estimation Task. Psychophysiology, 24(6):700-713.

[De Luca et al., 2006] De Luca, A., Albu-Schaeffer, A., Haddadin, S., and Hirzinger, G. (2006). Collision Detection and Safe Reaction with the DLR-III Lightweight Manipulator Arm. In Proceedings of the IEEE/RS International Conference on Intelligent Robots and Systems (IROS), pages $1623-1630$

[Dillenbourg et al., 1996] Dillenbourg, P., Baker, M., and Blaye, A. (1996). The evolution of research on collaborative learning. In Learning in Humans and Machine: Towards an interdisciplinary learning science., pages 189211.

[Dollar and Herr, 2008] Dollar, A. and Herr, H. (2008). Lower Extremity Exoskeletons and Active Orthoses: Challenges and State-of-the-Art. IEEE Transactions on Robotics, 24(1):144-158.

[Emken and Reinkensmeyer, 2005] Emken, J. L. and Reinkensmeyer, D. J (2005). Robot-enhanced motor learning: accelerating internal model formation during locomotion by transient dynamic amplification. IEEE Transactions on Neural Systems and Rehabilitation Engineering, 13(1):339.

[Evrard et al., 2009] Evrard, P., Gribovskaya, E., Calinon, S., Billard, A., and Kheddar, A. (2009). Teaching physical collaborative tasks: object-lifting case study with a humanoid. In Proceedings of the IEEE-RAS International Conference on Humanoid Robots, pages 399-404.

[Evrard and Kheddar, 2009a] Evrard, P. and Kheddar, A. (2009a) Homotopy-based controller for physical human-robot interaction. In Proceedings of the IEEE International Symposium on Robot and Human Interactive Communication, (RO-MAN), pages 1-6.

[Evrard and Kheddar, 2009b] Evrard, P. and Kheddar, A. (2009b). Homotopy switching model for dyad haptic interaction in physical collaborative tasks. In Proceedings of the IEEE EuroHaptics conference and Symposium on Haptic Interfaces for Virtual Environment and Teleoperator Systems, pages $45-50$.

[Franklin et al., 2008] Franklin, D., Burdet, E., Tee, K., Osu, R., Chew, C., Milner, T., and Kawato, M. (2008). CNS learns stable, accurate, and efficient movements using a simple algorithm. The Journal of Neuroscience, 28(44):11165-73.

[Franklin et al., 2003] Franklin, D. W., Osu, R., Burdet, E., Kawato, M., and Milner, T. E. (2003). Adaptation to stable and unstable dynamics achieved by combined impedance control and inverse dynamics model. Journal of Neurophysiology, 90(5):3270-3282.

[Gallese and Goldman, 1998] Gallese, V. and Goldman, A. (1998). Mirror neurons and the simulation theory of mind-reading. Trends in Cognitive Sciences, 2(12):493-501.

[Gentry, 2005] Gentry, S. (2005). Dancing cheek to cheek: Haptic communication between partner dancers and swing as a finite state machine. $\mathrm{PhD}$ thesis, Massachusetts Institute of Technology.

[Gibson et al., 1977] Gibson, J., Shaw, R., and Bransford, J. (1977). The Theory of Affordances. Lawrence Erlbaum, New Jersey.

[Gietelink et al., 2006] Gietelink, O., Ploeg, J., De Schutter, B., and Verhaegen, M. (2006). Development of advanced driver assistance systems with vehicle hardware-in-the-loop simulations. Vehicle System Dynamics, 44(7):569-590.

[Glynn et al., 2001] Glynn, S., Fekieta, R., and Henning, R. (2001). Use of Force-Feedback Joysticks to Promote Teamwork in Virtual Teleoperation. Proceedings of the Human Factors and Ergonomics Society Annual Meeting, 45(27):1911-1915.

[Groten et al., 2009] Groten, R., Feth, D., Goshy, H., Peer, A., Kenny, D., and Buss, M. (2009). Experimental analysis of dominance in haptic collaboration. In Proceedings of the IEEE International Symposium on Robot and Human Interactive Communication (RO-MAN), pages 723-729.

[Groten et al., 2010] Groten, R., Feth, D., and Peer, A. (2010). Shared decision making in a collaborative task with reciprocal haptic feedback-an efficiency-analysis. In Proceedings of the IEEE International Conference on Robotics \& Automation (ICRA), pages 1834-1839.

[Guanghui et al., 2011] Guanghui, Mina J, Deming W, and Hashimoto, M. (2011). Control for physical human-robot interaction based on online update of dynamics. In Proceedings of the IEEE International Conference on Computer Science and Automation Engineering (CSAE), volume 2, pages $280-284$.

[Haddadin et al., 2008] Haddadin, S., Albu-Schaffer, A., De Luca, A., and Hirzinger, G. (2008). Collision detection and reaction: A contribution to safe physical Human-Robot Interaction. Proceedings of the IEEE IEEE/RSJ International Conference on Intelligent Robots and Systems (IROS), pages 3356-3363.

[Hogan, 1985] Hogan, N. (1985). Impedance control: An approach to manipulation. In Proceedings of the American Control Conference, number March, pages 304-313.

[Hogan and Krebs, 2004] Hogan, N. and Krebs, H. I. (2004). Interactive robots for neuro-rehabilitation. Restorative Neurology and Neuroscience, 22(3-5):349-358

[Hokayem and Spong, 2006] Hokayem, P. and Spong, M. (2006). Bilateral teleoperation: An historical survey. Automatica, 42(12):2035-2057.

[Ikemoto et al., 2009] Ikemoto, S., Amor, B., Minato, T., Ishiguro, H., and Jung, B. (2009). Physical interaction learning: Behavior adaptation in cooperative human-robot tasks involving physical contact. In Proceedings of the International Symposium on Robot and Human Interactive Communication, (RO-MAN), pages 504-509.

[Jarrassé et al., 2012] Jarrassé, N., Charalambous, T., and Burdet, E. (2012). A framework to describe, analyze and generate interactive motor behaviors. PLoS ONE, 7(11):e49945.

[Kazerooni, 1990] Kazerooni, H. (1990). Human-robot interaction via the transfer of power and information signals. IEEE Transactions on Systems, Man and Cybernetics, 20(2):450-463.

[Kazerooni and Guo, 1993] Kazerooni, H. and Guo, J. (1993). Human Extenders. Journal of Dynamic Systems, Measurement, and Control, 115(2B):281-290.

[Kazerooni et al., 2005] Kazerooni, H., Racine, J., and Steger, R. (2005). On the Control of the Berkeley Lower Extremity Exoskeleton (BLEEX) 
Proceedings of the IEEE International Conference on Robotics and Automation (ICRA), pages 4353-4360.

[Kazerooni et al., 1986] Kazerooni, H., Sheridan, T., and Houpt, P. (1986). Robust compliant motion for manipulators. I: The fundamental concepts of compliant motion. II: Design method. IEEE Journal of Robotics and Automation, 2(2):83-105.

[Khatib, 1999] Khatib, O. (1999). Autonomous Systems Mobile manipulation : The robotic assistant. Robotics and Autonomous Systems, 26:175183.

[Kheddar, 2011] Kheddar, A. (2011). Human-robot haptic joint actions is an equal control-sharing approach possible? In Proceedings of the International Conference on Human System Interactions (HSI), pages 268273.

[Knoblich et al., 2010] Knoblich, G., Butterfill, S., and Sebanz, N. (2010). "Psychological research on joint action: Theory and data. Psychology of Learning and Motivation, 51:59-101.

[Kosuge and Hirata, 2004] Kosuge, K. and Hirata, Y. (2004). Human-Robot Interaction. Proceedings of the IEEE International Conference on Robotics and Biomimetics, pages 8-11.

[Kosuge et al., 1994] Kosuge, K., Yoshida, H., Taguchi, D., Fukuda, T. Hariki, K., Kanitani, K., and Sakai, M. (1994). Robot-human collaboration for new robotic applications. Proceedings of Annual Conference of IEEE Industrial Electronics, pages 713-718.

[Krüger et al., 2009] Krüger, J., Lien, T., and Verl, A. (2009). Cooperation of human and machines in assembly lines. CIRP Annals - Manufacturing Technology, 58(2):628-646

[Kwakkel et al., 2008] Kwakkel, G., Kollen, B. J., and Krebs, H. I. (2008) Effects of $\{$ Robot-Assisted $\}$ Therapy on Upper Limb Recovery After Stroke: A Systematic Review. Neurorehabilitation Neural Repair, 22(2):111-121.

[Lawitzky et al., 2010] Lawitzky, M., Mortl, A., and Hirche, S. (2010). Load sharing in human-robot cooperative manipulation. In Proceedings of the IEEE International Symposium on Robots and Human Interactive Communication (RO-MAN), pages 185-191.

[Lee et al., 2011] Lee, D., Ott, C., Nakamura, Y., and Hirzinger, G. (2011). Physical human robot interaction in imitation learning. In Proceedings of the IEEE International Conference on Robotics and Automation (ICRA), pages 3439-3440.

[Lum, 2002] Lum, P. (2002). Robot-assisted movement training compared with conventional therapy techniques for the rehabilitation of upperlimb motor function after stroke. Archives of Physical Medicine and Rehabilitation, 83(7):952-959.

[Maeda et al., 2001] Maeda, Y., Hara, T., and Arai, T. (2001). Humanrobot cooperative manipulation with motion estimation. In Proceedings of the IEEE/RSJ International Conference on Intelligent Robots and Systems (IROS), volume 4, pages 2240-2245.

[Marchal-Crespo and Reinkensmeyer, 2009] Marchal-Crespo, L. and Reinkensmeyer, D. (2009). Review of control strategies for robotic movement training after neurologic injury. Journal of NeuroEngineering and Rehabilitation, 6(1):20.

[Melendez-Calderon, 2011] Melendez-Calderon, A. (2011). Investigating sensory-motor interactions to shape rehabilitation. $\mathrm{PhD}$ thesis, Imperial College of Scence, Technology and Medicine. London.

[Melendez-Calderon et al., 2011] Melendez-Calderon, A., Komisar, V. Ganesh, G., and Burdet, E. (2011). Classification of strategies for disturbance attenuation in human-human collaborative tasks. In Proceedings of the International Conference of the IEEE Engineering in Medicine and Biology Society (EMBC), pages 2364-2367.

[Miller et al., 2000] Miller, B., Colgate, J., and Freeman, R. (2000). Guaranteed stability of haptic systems with nonlinear virtual environments. IEEE Transactions on Robotics and Automation, 16(6):712-719.

[Mortl et al., 2012] Mortl, a., Lawitzky, M., Kucukyilmaz, a., Sezgin, M., Basdogan, C., and Hirche, S. (2012). The Role of Roles: Physical Cooperation between Humans and Robots. The International Journal of Robotics Research.

[Myerson, 1997] Myerson, R. (1997). Game theory: analysis of conflict. Harvard University Press.

[Nudehi et al., 2005] Nudehi, S., Mukherjee, R., and Ghodoussi, M. (2005) A shared-control approach to haptic interface design for minimally invasive telesurgical training. IEEE Transactions on Control Systems Technology, 13(4):588-592.

[Obhi and Sebanz, 2011] Obhi, S. and Sebanz, N. (2011). Moving together: toward understanding the mechanisms of joint action. Experimental brain research., pages 329-336.

[Oguz et al., 2010] Oguz, S., Kucukyilmaz, A., Sezgin, T., and Basdogan, C. (2010). Haptic negotiation and role exchange for collaboration in virtual nvironments. In Proceedings of the IEEE Haptics Symposium, pages 371378

[Ong et al., 2008] Ong, K., Seet, G., and Sim, S. (2008). An implementation of seamless human-robot interaction for telerobotics. International Journal of Advanced Robotic Systems, 5(2):167-176.

[Passenberg et al., 2011a] Passenberg, C., Groten, R., Peer, A., and Buss, M. (2011a). Towards real-time haptic assistance adaptation optimizing task performance and human effort. In Proceedings of the World Haptics Conference (WHC), pages 155-160.

[Passenberg et al., 2010] Passenberg, C., Peer, A., and Buss, M. (2010). A survey of environment-, operator-, and task-adapted controllers for teleoperation systems. Mechatronics, 20(7):787-801.

[Passenberg et al., 2011b] Passenberg, C., Stefanov, N., Peer, A., and Buss, M. (2011b). Enhancing task classification in human-machine collaborative teleoperation systems by real-time evaluation of an agreement criterion. In Proceedings of World Haptics Conference (WHC), pages 493-498.

[Pastor et al., 2011] Pastor, P., Kalakrishnan, M., Chitta, S., and Theodorou, E. (2011). Skill Learning and Task Outcome Prediction for Manipulation. In Proceedings of the IEEE International Conference on Robotics and Automation (ICRA), pages 3828 - 3834 .

[Pentland and Liu, 1999] Pentland, A. and Liu, A. (1999). Modeling and Prediction of Human Behavior. Neural Computation, 11(1):229-242.

[Peshkin and Colgate, 2001] Peshkin, M. and Colgate, J. (2001). Cobot architecture. IEEE Transactions on Robotics and Automation, 17(4):377390.

[Pham et al., 2010] Pham, H., Ueha, R., Hirai, H., and Miyazaki, F. (2010). A study on dynamical role division in a crank-rotation task from the viewpoint of kinetics and muscle activity analysis. In Proceedings of the IEEE/RSJ International Conference on Intelligent Robots and Systems (IROS), pages 2188-2193.

[Premack and Woodruff, 1978] Premack, D. and Woodruff, G. (1978). Does the Chimpanzee Have a Theory of Mind? Behavioral and Brain Sciences, 1(04):515-526.

[Reed et al., 2007] Reed, K., Patton, J., and Peshkin, M. (2007). Replicating human-human physical interaction. In Proceedings ot the IEEE International Conference on Robotics and Automation (ICRA), number April, pages $3615-3620$.

[Reed and Peshkin, 2008] Reed, K. and Peshkin, M. (2008). Physical col laboration of human-human and human-robot teams. IEEE Transactions on Haptics, 1(2):108-120.

[Reed et al., 2006a] Reed, K., Peshkin, M., Hartmann, M., Patton, J., Vish ton, P., and Grabowecky, M. (2006a). Haptic cooperation between people, and between people and machines. In Proceedings of the IEEE/RSJ International Conference on Intelligent Robots and Systems (IROS), pages 2109-2114.

[Reed et al., 2006b] Reed, K., Peshkin, M., Hartmann, M. J., Grabowecky, M., Patton, J., and Vishton, P. M. (2006b). Haptically linked dyads: are two motor-control systems better than one? Psychological science, 17(5):365-

[Reinkensmeyer and Patton, 2009] Reinkensmeyer, D. J. and Patton, J. L. (2009). Can robots help the learning of skilled actions? Exercise and sport sciences reviews, 37(1):43-51.

[Richardson et al., 2007] Richardson, M., Marsh, K., Isenhower, R., Goodman, J., and Schmidt, R. (2007). Rocking together: dynamics of intentional and unintentional interpersonal coordination. Human Movement Science. 26(6):867-891.

[Rosenberg, 1993] Rosenberg, L. (1993). Virtual fixtures: Perceptual tools for telerobotic manipulation. Virtual Reality Annual International, pages $76-82$.

[Sakita et al., 2004] Sakita, K., Ogawam, K., Murakami, S., Kawamura, K., and Ikeuchi, K. (2004). Flexible cooperation between human and robot by interpreting human intention from gaze information. In In Proceedings of the IEEE/RSJ International Conference on Intelligent Robots and Systems (IROS), pages 846-851.

[Sallnas, 2001] Sallnas, E. (2001). Improved Precision in Mediated Collaborative Manipulation of Objects by Haptic Force Feedback. Haptic Human-Computer Interaction, pages 69-75.

[Santis et al., 2008] Santis, A., Siciliano, B., and De Luca, A. (2008). An atlas of physical human-robot interaction. Mechanism and Machine, 43:253-270.

[Sato et al., 1994] Sato, T., Nishida, Y., Ichikawa, J., Hatamura, Y., and Mizoguchi, H. (1994). Active understanding of human intention by a robot through monitoring of human behavior. Proceedings of IEEE/RS International Conference on Intelligent Robots and Systems (IROS'94), pages $405-414$

[Schaal et al., 1997] Schaal, S. et al. (1997). Learning from demonstration. Advances in neural information processing systems, pages 1040-1046. 
[Schraft et al., 2005] Schraft, R. D., Meyer, C., Parlitz, C., and Helms, E. (2005). PowerMate A Safe and Intuitive Robot Assistant for Handling and Assembly Tasks. In Proceedings of the IEEE International Conference on Robotics \& Automation (ICRA), pages 4074- 4079.

[Schütz-Bosbach and Prinz, 2007] Schütz-Bosbach, S. and Prinz, W. (2007) Perceptual resonance: action-induced modulation of perception. Trends in cognitive sciences, 11(8):349-55.

[Sebanz et al., 2006] Sebanz, N., Bekkering, H., and Knoblich, G. (2006). Joint action: bodies and minds moving together. Trends in Cognitive Sciences, 10(2):70-76.

[Sebanz et al., 2003] Sebanz, N., Knoblich, G., and Prinz, W. (2003). Representing others' actions: just like one's own? Cognition, 88(3):B11-B21.

[Sheridan, 1992] Sheridan, T. (1992). Telerobotics, automation, and human supervisory control. The MIT press.

[Sheridan, 1997] Sheridan, T. (1997). Eight ultimate challenges of humanrobot communication. Proceedings of the IEEE International Symposium on Robot and Human Interactive Communication, (RO-MAN), pages 9-14.

[Stefanov et al., 2009] Stefanov, N., Peer, A., and Buss, M. (2009). Role determination in human-human interaction. World Haptics, pages 51-56.

[Stückler and S Behnke, 2011] Stückler, J. and S Behnke (2011). Following human guidance to cooperatively carry a large object. In Proceedings of the 11th IEEE-RAS International Conference on Humanoid Robots (Humanoids), pages 218-223.

[Surdilovic et al., 2001] Surdilovic, D., Grassini, F., and De Bartolomei, M. (2001). Synthesis of impedance control for complex co-operating robot assembly task. In Proceedings of the IEEE/ASME International Conference on Advanced Intelligent Mechatronics (AIM), volume 2, pages 1181-1186 vol.2. IEEE.

[Ueha et al., 2009] Ueha, R., Pham, H., Hirai, H., and Miyazaki, F. (2009). A simple control design for human-robot coordination based on the knowledge of dynamical role division. In Proceedings of the IEEE/RSJ International Conference on Intelligent Robots and Systems (IROS), pages 3051-3056.

[Vertegaal et al., 2001] Vertegaal, R., Slagter, R., van der Veer, G., and Nijholt, A. (2001). Eye gaze patterns in conversations: there is more to conversational agents than meets the eyes. In Proceedings of Conference on Human Factors in Computing Systems (SIGCHI), CHI '01, pages 301308.

[Wang et al., 2009] Wang, Z., Peer, A., and Buss, M. (2009). An HMM approach to realistic haptic human-robot interaction. In Proceedings of the EuroHaptics conference and Symposium on Haptic Interfaces for Virtual Environment and Teleoperator Systems., pages 374-379.

[Wojtara et al., 2009] Wojtara, T., Uchihara, M., Murayama, H., Shimoda, S., Sakai, S., Fujimoto, H., and Kimura, H. (2009). Humanrobot collaboration in precise positioning of a three-dimensional object. Automatica, 45(2):333-342.

[Yanco and Drury, 2002] Yanco, H. and Drury, J. (2002). A taxonomy for human-robot interaction. In Proceedings of the AAAI Fall Symposium on Human-Robot Interaction, pages 111-119.

[Yanco and Drury, 2004] Yanco, H. and Drury, J. (2004). Classifying humanrobot interaction: an updated taxonomy. Proceedings of the IEEE International Conference on Systems, Man and Cybernetics, pages 2841-2846.

[Yang et al., 2011] Yang, C., Ganesh, G., Haddadin, S., Parusel, S., AlbuSchaeffer, A., and Burdet, E. (2011). Human-like Adaptation of Force and Impedance in Stable and Unstable Interactions. IEEE Transaction on Robotics, 27(5):1-12.

[Yokoyama et al., 2003] Yokoyama, K., Handa, H., Isozumi, T., Fukase, Y., Kaneko, K., Kanehiro, F., Kawai, Y., Tomita, F., and Hirukawa, H. (2003). Cooperative works by a human and a humanoid robot. In Robotics and Automation, 2003. Proceedings. ICRA'O3. IEEE International Conference on, volume 3, pages 2985-2991. IEEE.

[Zajonc, 1965] Zajonc, R. (1965). Social facilitation. Science, 149(3681):269-274.

[Zeng et al., 2009] Zeng, Q., Burdet, E., and Teo, C. (2009). Evaluation of a collaborative wheelchair system in cerebral palsy and traumatic brain injury users. Neurorehabilitation and Neural Repair, 23(5):494. 